\title{
Der Erziehungswahn und sein Sinn (Nietzsche)
}

I.

Die Forderung "Mehr Mut zur Erziehung! « ist inzwischen topisch. ${ }^{1}$ Mit ihrer Implikation, Erziehung sei zum Fürchten, stellt sie Erziehung als eine Machtausübung hin, die sich nicht unbedingt lauterer Mittel bedient und deren Inanspruchnahme folglich der mutigen Entschlossenheit bedarf, für die das Sprichwort "wo gehobelt wird, fallen Späne« auf seine Weise wirbt. In der Mut-Formel steckt mithin ein verkapptes "Lob der Disziplin «"; gelobt werden kann die Disziplin, zur erzieherischen Zucht eigens zu ermutigen ist aber nur, weil diese nicht bloß Zöglinge, sondern offenbar auch die Erzieher das Fürchten lehrt. Die Vorstellung, zur Erziehung bedürfe es heldenhafter Selbstüberwindung, ist nicht auf traditionellen Werte-Konservatismus beschränkbar. Auch Vertreter eines anti-autoritären Erziehungskonzeptes werden Mut zu ihrer Form von Erziehung fordern (müssen), da sie sonst keine Erzieher, sondern Nicht-Erzieher wären, und anti-autoritäre Erziehung nicht als pädagogische Alternative zu herkömmlicher Disziplinierung antreten könnte. Erziehung scheint zur Herrschaft verdammt, denn jedes Erziehungsmodell muß von seiner Mächtigkeit überzeugt sein. Deshalb bietet sich als ein erster Schritt zur Entmachtung der Erziehung deren Unterschätzung an. ${ }^{3}$

$\mathrm{Daß}$ dies eine Zumutung darstellt, zeigt die Reaktion vieler Pädagogen auf entsprechende Vorschläge jüngerer Theoriebildung, wie sie beispielsweise

1 Anzunehmen ist, daß der Ursprung der vor allem in bildungspolitischen Kontexten und in der Ratgeberliteratur heimischen Formel auf die gleichnamige Tagung datiert, die 1978 am Wissenschaftszentrum in Bonn-Bad Godesberg stattfand. Während der programmatische Titel in den 70er Jahren als konservatives Fanal gegen die anti-autoritäre Erziehung galt, ist eine derart eindeutige politische Zurechnung heute kaum noch gegeben.

$2 \quad$ So der Titel des jüngsten erzieherischen Vorstoßes des Salemer Internatsleiters Bernhard Bueb, Lob der Disziplin. Eine Streitschrift, Berlin 2006.

3 Bei diesem Versuch hat man vom Appell an alternative Machtinstanzen wie etwa sdie Gene oder die speer group abzusehen, da in diesem Fall die furchterregende Macht der Erziehung nur durch eine andere, ebenso wirkmächtige und schlechterdings nicht zu überschätzende Macht ersetzt würde. 
mit Michel Foucaults Studien zur Disziplinarmacht vorliegen. ${ }^{4}$ Der selbstkritischen Auskunft einiger Wissenschaftler zufolge ist Foucaults problematischer Status in ihrem Fach den Konsequenzen seiner Analysen für die pädagogische Leitdifferenz von Heteronomie und Autonomie geschuldet. ${ }^{5}$ Foucaults Aufweis der grundlegenden Ambivalenz aller Subjektivierungsprozesse $^{6}$ erzwinge den Abschied von der "Pathosformel Mündigkeit « ${ }^{7}$, die als ultimativer Zweck jene Mittel heiligen soll, die sich mit dem Autonomiepostulat moderner Pädagogik nicht vertragen. Kant hat den hier waltenden Grundwiderspruch moderner Erziehung bündig formuliert: »Eines der größten Probleme der Erziehung ist, wie man die Unterweisung unter den gesetzlichen Zwang mit der Fähigkeit sich seiner Freiheit zu bedienen, vereinigen könne. Denn Zwang ist nöthig! Wie cultiviere ich die Freiheit bei dem Zwange? ${ }^{8}$ Daß Foucault an pädagogische Dogmen rührt, macht ihn aber noch nicht zu einem Unterschätzer von Erziehung. Mit der These, daß Subjektwerdung prinzipiell Unterwerfung beinhalte, mutet auch Foucault Erziehungsoperationen erstaunlich viel Verantwortung für die Menschwerdung des Menschenkindes zu. Das Skandalon Foucaults besteht folglich nicht im Zweifel an der Mächtigkeit der Erziehung, sondern in seiner Umformulierung des Machtbegriffs. Die von Foucault so genannte Disziplinarmacht läßt sich im Unterschied zum älteren Souveränitätsparadigma keiner personalen Autorität mehr zuschreiben. So entmachtet Disziplinarmacht zwar den einzelnen Erzieher-Souverän, aber die nun diffus verteilte und auf Verinnerlichung setzende Macht ist bekanntlich kaum weniger furchterregend als vormoderne Erziehungszucht. ${ }^{9}$

Vergleichsweise allergischer reagiert man hingegen auf die "Irritationen des Erziehungssystems $\aleph^{10}$ durch die Systemtheorie. Dirk Baecker hat darauf hin-

4 Vgl. Michel Foucault, Überwachen und Strafen. Die Geburt des Gefängnisses, Frankfurt a.M. 1977.

5 Vgl. Markus Rieger-Ladich/Norbert Ricken (Hg.), Michel Foucault. Pädagogische Lektüren, Wiesbaden 2004 sowie Käte Meyer-Drawe, Illusionen von Autonomie. Diesseits von Ohnmacht und Allmacht des Ich, München ${ }^{2} 2000$.

6 Vgl. im Ausgang von Foucault auch Judith Butlers Überlegungen zur Genese des Gewissens, in: Dies., The Psychic Life of Power. Theories in Subjection, Stanford 1997.

7 Markus Rieger-Ladich, Mündigkeit als Pathosformel. Überlegungen zur pädagogischen Semantik, Konstanz 2002.

8 Immanuel Kant, Über Pädagogik, in: Ders., Werke, Akademieausgabe, Bd. IX, Berlin 1968, S. 441-499, hier S. 453.

9 Vgl. Katharina Rutschky (Hg.), Schwarze Pädagogik. Quellen zur Naturgeschichte der bürgerlichen Erziehung, Frankfurt a.M./Berlin 1988.

10 Dieter Lenzen (Hg.), Irritationen des Erziehungssystems. Pädagogische Resonanzen auf Niklas Luhmann, Frankfurt a.M. 2004. 
gewiesen, daß Luhmann in einer Art vorauseilender Rücksichtnahme auf die Empfindlichkeiten der pädagogischen Wissenschaft sein Erziehungsbuch nicht, wie es die Reihe der anderen Abhandlungen nahegelegt hätte, die Erziehung der Gesellschaft genannt hat, sondern das "Erziehungssystem der Gesellschaft «. ${ }^{11}$ Derlei Rücksichten nimmt Baecker nicht. In seiner an prominentem Ort in der taz publizierten Kleinen Soziologie der Erziehung wirft er dem Erziehungssystem vor, den historisch fälligen Grad der Ausdifferenzierung bisher schuldig geblieben zu sein. Doppelzüngig heißt es im ersten von insgesamt sechs Beiträgen: "Was ist in der Erziehung schief gelaufen? Warum fehlt ihr eine Kritik, die in der modernen Gesellschaft der Beleg einer gelungenen Ausdifferenzierung ist? $\ll^{12}$ Schuld an diesem Mangel trägt in Baeckers Sicht die notorische Selbstüberschätzung der Erziehung. Zu deren Symptomen gehöre insbesondere, daß sich die Erziehung bisher entweder im Blick auf Macht oder auf Liebe (was ja in gewisser Weise auf dasselbe hinausläuft) über sich selbst verständigt hat. Den von der Systemtheorie anfangs favorisierten alternativen Austauschmedien Kind und Lebenslauf fügt Baecker in direktem Anschluß an Parson Intelligenz hinzu. ${ }^{13}$ Die Umstellung von Kind bzw. Lebenslauf auf Intelligenz scheint Baecker geeignet, das dringlich eingeklagte Bewußtsein der eigenen Grenzen vorzubereiten. Baecker nennt dies "Limitationalität« und drängt in ihrem Namen auch auf eine andere Gewichtung der Leitdifferenz Wissen vs. Nichtwissen zugunsten des letzteren. ${ }^{14}$ Entsprechend wäre auch die Unterscheidung reif/unreif zu ersetzen durch diejenige von kreativ/routiniert. Die systemtheoretische Fähigkeit, von allen Inhalten zu abstrahieren, schlägt in einem Erziehungsmodell zu Buche, das die Gegenstände (des Wissens oder Lernens) ebenso wie die Verfahren bewußt und programmatisch unterschätzt.

Systemtheoretisch fundierte Vorschläge zur Umorganisation des Erziehungssystems können sich solche Unterschätzung leisten, weil sie nicht Autonomie, sondern Effizienz und Flexibilität zum Ziel haben. Im Unterschied zur bildungsbürgerlich-liberalen Pädagogik, aber auch im Unterschied zur Disziplinierungsrhetorik, die nicht in ihren Zielen, sondern in ihren Mitteln divergieren, erweist sich die systemtheoretische Pädagogik als ideal zuge-

11 Niklas Luhmann, Das Erziehungssystem der Gesellschaft, Frankfurt a.M. 2002.

12 Dirk Baecker, Kleine Soziologie der Erziehung. Sechsteilige Serie in der »taz«, Februar/ März 2004.

13 Ders., Erziehung im Medium der Intelligenz, http://homepage.mac.corn/baecker/, S. 1-45.

14 Baeckers Plädoyer für die Dummheit hat in Rancieres Studie zu Jacotot einen ihm wohl unbekannten Vorläufer, vgl. Jacques Rancière, The Ignorant Schoolmaster, Five Lessons in Intellectual Emancipation, New York 1991. 
schnitten auf die Anforderungen und Herausforderungen in modernen Wissensgesellschaften. Inhaltsunabhängige Flexibilität macht tauglich für die Navigation auf den Märkten der new economy; Limitationalität ist nicht zufällig ein Begriff aus der Wirtschaftssprache. Die von der Systemtheorie geleistete theoretische Nobilitierung der sich selbst regulierenden Anpassung wird in Erziehungspraktiken übersetzt, die die seit dem Bologna-Prozeß immer spürbarere Amalgamierung von Erziehungsdiskursen mit ökonomischen Modellen, Begriffen und Zielen kongenial vertiefen.

Mit kritischer Sorge haben Jan Masschelein und Maarten Simons den Einzug ökonomischer Nomenklatur in die Rede über Erziehung beobachtet. ${ }^{15}$ Im Ausgang von Foucault versuchen sie dessen Modell der Disziplinarmacht unter veränderten gesellschaftlichen Bedingungen weiterzudenken und warnen, daß die gepriesene unternehmerische Einstellung zum eigenen Selbst die Erfahrung einer potentiell kritisch-resistenten Unproduktivität und Ineffizienz (die früher einmal mit Kreativität assoziiert wurden) systematisch blockiere. In ihrer Studie erscheint das sich gegenwärtig reformierende Erziehungssystem als besonders perfider Agent einer ideal angepaßten Macht. Systemtheoretiker können solche Befürchtungen nur belächeln, denn sie wissen, daß auch und gerade systemkritische Positionen im Dienst der allgemeinen Anpassung des Erziehungssystems an die Erfordernisse der jeweiligen gesellschaftlich fälligen Transformationsprozesse stehen. Das war schon die Pointe von Parsons einschlägiger Beschreibung der US-amerikanischen Universitätsunruhen in den späten 60er Jahren. Auch damals vollzog sich der eigentliche Sinn und Zweck der Universitätskritik, nämlich die Anpassung des Systems, im Rücken der protestierenden Akteure. ${ }^{16}$ Dieses Absehen von Inhalten und Intentionen exponiert sowohl die theoretische Superiorität der Systemtheorie als auch ihren altmodisch besserwisserischen Zug, denn ob nun so oder so entschieden worden sein wird, immer wird es der evolutionären Anpassung gesellschaftlicher Systeme an sich ändernde Verhältnisse gedient haben. Man braucht die Pathosformel Mündigkeit nicht zu beschwören, um in dieser Entmachtung sich in Bildungsdebatten kritisch positionierender Subjekte einen problematischen Effekt der systemtheoretischen Unterschätzung von Erziehung zu erkennen. Deshalb ist nach ande-

15 Jan Masschelein/Maarten Simons, Globale Immunität oder Eine kleine Kartographie des europäischen Bildungsraums, Zürich/Berlin 2005.

16 Vgl. Talcott Parsons und Gerald M. Platts Beschreibung der Bildungsdebatten der 68er in: Dies., Die amerikanische Universität. Ein Beitrag zur Soziologie der Erkenntnis, übers. von Michael Bischoff, Frankfurt a.M. 1990. 
ren Möglichkeiten zu fragen, die Erziehung von ihrer Mut heischenden Macht und beängstigenden Verantwortungsbürde zu entlasten. Eine dieser Möglichkeiten wird im folgenden anhand von Nietzsche spekulativ erprobt. Nietzsche bietet sich in diesem Zusammenhang nicht zuletzt aufgrund seiner historischen Schwellenposition und entsprechender Ambivalenzen an. Gilt er einerseits als Klassiker der Kritik an der bürgerlichen Bildung, so scheinen seine Zucht- und Züchtungsphantasmen andererseits den auf aller Erziehung lastenden machtpolitischen Verdacht zu radikalisieren.

II.

Die Macht der guten oder schlechten Gewissens als Herrschaftspraxis begriffenen Erziehung stand seit der Inauguration des humanistischen Bildungsideals in problematischen Beziehungen zur anderen Macht im Staate, dem Staate selbst. Der Begründer der modernen Universität Friedrich Wilhelm Humboldt gab seinen Posten als Direktor der später nach ihm benannten Berliner Universität bekanntlich kurze Zeit nach Amtsantritt wegen Konflikten mit dem Staat auf. ${ }^{17}$ In dem Maße, in dem der moderne Staat auf die Monopolisierung aller Gewaltausübung drängt und dieses Ziel mit Hilfe der Unterscheidung zwischen rechtsförmiger und unrechtmäßiger Gewalt verfolgt, fallen Bildung und Erziehung zwangsläufig in seinen Einzugs- und Einflußbereich. Davon zeugen nicht nur die Debatten um eine Bildungs- bzw. Kulturpolizei im 19. Jahrhundert ${ }^{18}$, sondern auch die vor allem seit Beginn des 20. Jahrhunderts rasch fortschreitende Verrechtlichung der ehemals als Naturzwecke zur Gewaltanwendung freigegebenen Erziehungsabsichten Einzelner wie z.B. Eltern oder Lehrer. Die bei den Entscheidungen im Rahmen der Exzellenzinitiative letztes Jahr aufgetretenen, aber rasch beigelegten Zwistigkeiten zwischen Politik und Wissenschaft haben jüngst noch einmal an den alten Machtkampf zwischen Staat und Bildung erinnert. Aber kaum jemand wird heute allen Ernstes noch die These von der Erziehung als Reproduktionsmedium des Staatsapparates vertreten wollen, wie sie einst von Louis Althusser oder Siegfried Bernfeld formuliert

17 Vgl. Käte Meyer-Drawe, Erziehung und macht, in: Vierteljahrsschrift für wissenschaftliche Pädagogik 77/4 (2001), S. 446-457.

18 Vgl. Robert von Mohl, Die Polizeiwissenschaft nach den Grundsätzen des Rechtsstaats, Tübingen 1833, I, S. $408 f f$. 
wurde. ${ }^{19}$ Mit den jüngsten Privatisierungsbestrebungen im Bildungssektor scheint die Epoche der "staatlichen Bewirtschaftung des Geistes" endgültig zu Ende zu gehen. Im Namen des Hochschulfreiheitsgesetzes schickt sich derzeit ein Staat an, die Bildung sich selbst zu überlassen. Die Freiheit, derer fortan die Hochschulen sich erfreuen müssen, kommt allerdings wegen mangelnder Ressourcen eher einer Verbannung gleich. Vor diesem aktuellen Hintergrund erscheinen Positionen wie diejenige Althussers rückblickend als mutwillige Zerstörung eines der wenigen verbliebenen Schutz- und Freiräume. Erst heute ahnt man, daß die Hoheit des Staates (bzw. der Staaten) über Bildungsfragen auch eine Entlastung der Bildungsstätten von den ihnen offenbar unwiderruflich einbeschriebenen Konflikten zwischen Mitteln und Zwecken der Erziehung bedeutet haben könnte.

Mit der Universität als staatsgeschützter Sphäre ist es jedenfalls fortan vorbei. Aber die Globalisierung rückt andere Verdächtigungs- und folglich auch Überschätzungsmöglichkeiten der Erziehung und ihrer Macht in den Blick. Erziehung steht nicht nur im Verdacht, der fortschreitenden Ökonomisierung zuzuarbeiten, sondern es gibt auch Anlaß zu der Vermutung, sie sei eine Form von Biomacht im Sinne Foucaults oder auch Agambens, die nacktes Leben produziert, um es sich zu unterwerfen.

Bereits Kant schied 1803 in seinen Vorlesungen zur Pädagogik die negativen, bloß zähmenden, das Rohe und Wilde abschleifenden Aspekte der Erziehung von den positiven und bildenden. Noch Adorno spricht unumwunden von der Notwendigkeit der »Entbarbarisierung ${ }^{20}{ }^{20}$ Diese in vielfältigen Schattierungen wiederkehrende und auf die vormoderne Differenz von educatio als primärer Sozialisation und institutio als Unterweisung bzw. Unterricht zurückgehende Unterscheidung steht schon bei Kant im Dienst der Entparadoxalisierung des Urwiderspruchs aller modernen Erziehung: Erziehung zur Autonomie sieht sich gezwungen, zunächst ein natürliches, unmündiges Leben setzend vorauszusetzen, aus dem herauszuführen sie sodann zu ihrer eigenen Aufgabe erklären kann. Mit Rekurs auf Agambens Überlegungen in seiner Studie zum homo sacer kann man argumentieren, daß

19 Vgl. Louis Althusser, Marxismus und Ideologie, Westberlin 1973. Siegfried Bernfeld, Sysiphos oder die Grenzen der Erziehung, Frankfurt a.M. 1973 (1925). Natürlich verabschiedet sich ineins damit auch die Vorstellung von Erziehung als Königsweg zu neuen gesellschaftlichen Verhältnissen, an die vor allem die Linke glaubte. Bildung ist kulturelles Kapital und Erziehung eine Ressource.

20 Vgl. Theodor W. Adorno, Erziehung zur Mündigkeit. Vorträge und Gespräche mit Hellmut Becker, 1959-1969, hg. von Gerd Kadelbach, Frankfurt a.M. 1970, S. $137 f f$. 
diese Unterscheidung eine Art Urszene der erzieherischen Selbstermächtigung darstellt. ${ }^{21}$ Ein bloßes, nacktes, wildes und zu zähmendes Leben wird im Akt seiner Ausgrenzung produziert, um dieses Leben dann den Techniken der Zucht unterwerfen zu können. ${ }^{22}$

Dieser Verdacht scheint zunächst absurd, denn im Zeichen moderner Biopolitik bekommt Erziehung in Gestalt der neuen Wissenschaft der Eugenik am Ende des 19. Jahrhunderts erstmals Konkurrenz bei der Herstellung individueller und kollektiver Menschenkörper. Zu dem Zeitpunkt, da die zunehmende Verrechtlichung von Erziehungsprozessen in der Reformpädagogik und der Jugendbewegung Versuche zeitigt, die Erziehung den sie verwaltenden Instanzen zu entziehen, treten Erziehung und Züchtung semantisch auseinander. Um 1900 zerfällt das alte begriffsgeschichtliche Kontinuum, auf dem jahrhundertelang sowohl Erziehung als auch Züchtung im Rahmen des übergreifenden Begriffs der Zucht Platz fanden. ${ }^{23}$ Die Unterscheidung von Züchtung und Erziehung verdeckt aber gerade jenen uralten Zusammenhang, der in den Totalitarismen des 20. Jahrhunderts eine Radikalisierung erfuhr, die es im Rückblick nur zu ratsam erscheinen läßt, Erziehung von Individuen und Züchtung von Volkskörpern kategorisch zu trennen. Ihrem eigenen Selbstverständnis nach war aber die Nationalpädagogik keineswegs eine Alternative, sondern ein Komplement der Eugenik; die Rede vom neuen Menschen trieb Pädagogen und Eugeniker gleichermaßen $u^{24}$, und schon Kant witterte hinter der Edukation das große Geheimnis der Vervollkommnung der Spezies Mensch. ${ }^{25}$ Zur Vergegenwärtigung des Zusammenhangs von Züchtung und Erziehung reicht ein Blick auf das Haustier. Die Zähmbarkeit von Haustieren ruht nicht allein auf individueller Erziehung, sondern deren Möglichkeit wird durch züchterische Inter-

21 Zu diesem Gedankenexperiment vgl. Verf.: Erziehung als blinder Fleck bei Agamben, in: Texte zur Kunst 53 (2004), S. 104-113. Vgl. auch Käte Meyer-Drawe, Erziehung und Macht, a.a.O.

22 Für Agamben ist diese biopolitische Dimension aber nicht wie für Foucault und andere eine Transformation der alten Disziplinarmacht, sondern die Offenbarung des Geheimnisses aller Souveränität. Vgl. Giorgio Agamben, Homo Sacer. Die souveräne Macht und das nackte Leben, aus dem Italienischen von Hubert Thüring, Frankfurt a.M. 2002.

23 Vgl. Grimms Wörterbuch sowie Petra Gehrings Kapitel über Züchtung, in: Was heißt Biopolitik?, Frankfurt a.M. 2006, S. 154-183.

24 Vgl. Ulrich Hermann, Neue Erziehung. Neue Menschen. Erziehung und Bildung zwischen Kaiserreich und Diktatur, Weinheim/Basel 1987.

25 »Denn hinter der Education steht das große Geheimnis der Vervollkommnung des Menschen.« Immanuel Kant, Über Pädagogik, S. 441. 
vention unterstützt. Immerhin nannte Kant den Menschen ein Tier, das erzogen werden muß. ${ }^{26}$

Unter skandalumwitterten Umständen hat vor einiger Zeit Peter Sloterdijk an die Zusammenhänge von Zähmung und Züchtung im Ausgang von Platon erinnert, dessen pädagogische Vorstellungen im Rahmen seiner Staatstheorie züchterische Praktiken wie selbstverständlich einschlossen. ${ }^{27}$ Sloterdijk behauptete in seinem Essay Regeln für den Menschenpark, "daß die Domestikation des Menschen das große Ungedachte ist, vor dem der Humanismus von der Antike bis zur Gegenwart die Augen abwandte«. ${ }^{28}$ In diesem $\mathrm{Zu}$ sammenhang komme Nietzsche das einzigartige Privileg zu, an das sich hier verbergende Domestikationsgeheimnis immerhin gerührt zu haben: „Er nimmt $\mathrm{Maß}$ an den zurückliegenden tausendjährigen Prozessen, in denen bisher dank intimer Verschränkungen von Züchtung, Zähmung und Erziehung Menschenproduktion betrieben wurde «. ${ }^{29}$

Nietzsches Werk ist auf derselben historischen Schwelle anzusiedeln, an der sich die Wege von Züchtung und Erziehung begriffsgeschichtlich trennen. Zwar bezeugt Nietzsches terminologische Praxis, Erziehung und Zähmung, Zucht und Züchtung häufig synonym zu verwenden, noch die Beharrungskräfte des alten semantischen Kontinuums, aber in der Sache scheint es doch schon um zwei sehr verschiedene Dinge zu gehen. Nietzsche kämpft einerseits noch auf der Front des alten Bildungsideals gegen den Staat, aber er propagiert andererseits auch schon individuelle und kollektive "Höherzüchtung«. Die Basler Vorträge zur Zukunft unserer Bildungsanstalten sind das früheste und nachhaltigste Dokument einer Bildungskritik, die noch ganz in den Bahnen der Auseinandersetzung zwischen Staat und Bildung ver-

26 Ebd.

27 Vgl. Werner Jaeger, Paideia. Die Formung des griechischen Menschen, Berlin/New York 1973, S. 840-846.

28 Peter Sloterdijk, Regeln für den Menschenpark. Ein Antwortschreiben zu Heideggers Brief über den Humanismus, Frankfurt a.M. 1999, S. 43. Und tatsächlich dürfte der gegenwärtig immer lauter werdende Ruf nach Zucht und Disziplin in den überalterten westlichen Gesellschaften auch mit der Wahrnehmung einer zunehmenden »Verwilderung« der Jugend zu tun haben, deren Bilder jüngst aus Pariser Vorstädten, zuvor aus Los Angeles zu sehen waren. Vor allem aber sind es "entfesselte Horden" Jugendlicher aus den arabischen und afrikanischen Ländern, die die Furcht vor wild gewordenen Un-Erzogenen schüren. Sloterdijks jüngstes Buch: Zorn und Zeit. Politisch-Psychologischer Versuch, Frankfurt a.M. 2006 nimmt sich dieses Themas an und führt damit Überlegungen fort, die Hans Magnus Enzensberger in seinem Essay: Aussichten auf den Bürgerkrieg, Frankfurt a.M. 1993, begonnen hatte.

29 Peter Sloterdijk, Regeln, S. 41. 
läuft. ${ }^{30} \mathrm{Zu}$ ihrem unmittelbaren Entstehungshintergrund gehören die Falkschen Reformen der Volksschulen. Wie Lagarde und das katholische Lager geißelte auch Nietzsche diesen Vorstoß des Staates, die Kirchen von ihrer Trägerschaft zu entbinden, als usurpatorische Geste. Daß Nietzsche Staat und Bildung für schlechterdings inkompatibel hielt, zeigt, wie sehr er sich dem humanistischen Bildungsideal noch verpflichtet wußte. Sarkastisch formulierte er in Menschliches, Allzumenschliches: „Die Regierungen der grossen Staaten haben zwei Mittel in den Händen, das Volk von sich abhängig zu erhalten [...] ein gröberes, das Heer, ein feineres, die Schule «. ${ }^{31}$ Was Nietzsche dagegen aufbietet, erschöpft sich aber doch nicht in einer utopistischen Rehabilitation des neuhumanistischen Bildungsideals, sondern greift dieses selbst an. ${ }^{32}$ Den Grundwiderspruch der Erziehung zwischen Mitteln und Zwecken betrachtend, müssen ihm alle Formen der Erziehung zwangsläufig als eine sich verleugnende und deshalb verlogene Machtausübung erscheinen: "Es giebt keine Erzieher. - Nur von Selbst-Erziehung sollte man als Denker reden. Die Jugend-Erziehung ist entweder ein Experiment, an einem noch Unerkannten, Unerkennbaren vollzogen, oder eine grundsätzliche Nivellierung, um das neue Wesen, welches es auch sei, den Gewohnheiten und Sitten, welche herrschen gemäss zu machen ${ }^{33}{ }^{33}$ Die "Sclaverei der Bildung und Erziehung « ${ }^{34}$ fängt folglich nicht erst in der Schule an: »keine Mutter zweifelt im Grunde ihres Herzens daran, am Kinde sich ein Eigenthum geboren zu haben, kein Vater bestreitet sich das Recht, es seinen Begriffen und Werthschätzungen unterwerfen zu dürfen « ${ }^{35}$ Aus seiner derart massiven und prinzipiellen Kritik zog Nietzsche zunächst den Schluß, daß alle höhere Erziehung nur der Ausnahme, dem Genie vorbehalten sein sollte. ${ }^{36}$ Im Möglichkeitsbereich seiner Kritik liegt implizit aber auch die Konsequenz, Erziehung, die etwas anderes sein will oder zu sein behauptet

30 Vgl. u.a. Gerhart Schmidt, Nietzsches Bildungskritik, in: Mihailo Djuric/Josef Simon (Hg.), Zur Aktualität Nietzsches, Würzburg 1984, S. 7-16.

31 Friedrich Nietzsche, Menschliches, Allzumenschliches II, in: Ders., Kritische Studienausgabe in 15 Bden., hg. Giorgio Colli und Mazzino Montinari, Berlin/New York 1980, Bd. 2, S. 509; vgl. auch den Passus über »Die Entwicklung des Geistes vom Staate gefürchtet", ebd., S. $308 f$.

32 Vgl. aber die Einschätzung Peter Uwe Hohendahls in: Ders., Literarische Kultur im Zeitalter des Liberalismus 1830-1870, München 1985, S. 274-279 und S. 381-388.

33 Nietzsche, Menschliches, Allzumenschliches II, S. 667f.

34 Ders., Ueber die Zukunft unserer Bildungsanstalten (Vortrag III), in: Ders., KSA 1, S. 739.

35 Ders., Jenseits von Gut und Böse, in: Ders., KSA 5, S. 116.

36 Vgl. die Schlußpassagen des letzten Vortrags, in: Ders., Ueber die Zukunft unserer Bildungsanstalten, in: Ders., KSA 1, S. $751 f$. 
als schiere Macht, schlechterdings in Abrede zu stellen. Eine Erfüllung des humanistischen Bildungsideals gibt es nicht nur nicht, weil die Umstände, Staat und Kirche, Fürsten und Väter es nicht dulden, sondern es kann sie nicht geben, weil alle Erziehung in Machtausübung verstrickt und deshalb widersprüchlich bleibt. Jede Erziehung bedarf der pia fraus, welche die Menschheitsverbesserer von jeher kannte und einsetzten: "Die Moral der Züchtung und die Moral der Zähmung sind in den Mitteln, sich durchzusetzen, vollkommen einander würdig«, denn beide müssen den Grundwiderspruch aller Erziehung befolgen, »dass, um Moral zu machen, man den unbedingten Willen zum Gegentheil haben muss. [...] In Formel ausgedrückt, dürfte man sagen: alle Mittel, wodurch bisher die Menschheit moralisch gemacht werden sollte, waren von Grund aus unmoralisch «. ${ }^{37}$ Gegen den Schluß, Erziehung und Höherzüchtung seien unmöglich, spricht nicht nur Nietzsches Pathos der Ausnahmefiguren, sondern gegen ihn sprechen vor allem seine Züchtungsvisionen. Der gegen Verhaustierung und Kleinzüchtung des Menschen predigende Zarathustra ist selbst ein Lehrer und Erzieher. Daß Züchtung keine Alternative zur Erziehung, sondern eine alternative Erziehung darstellt, bezeugt mehr als jeder andere Text das nachgelassene Anti-Darwin-Fragment, das Gerd Schank mit Recht zur Grundlage seiner Studie über die Begriffe Rasse und Züchtung bei Nietzsche gemacht hat. ${ }^{38}$ Ungeachtet seiner späteren Rezeption hat der von Nietzsche dort zugrundegelegte Züchtungsbegriff nichts mit zeitgenössischer Eugenik zu tun ${ }^{39}$, denn einerseits hält Nietzsche die Erblichkeit für zu »capriciös«, andererseits ist ihm das Genie als wahres Ziel aller Züchtung und Erziehung ein Phänomen sui generis: "dergleichen vererbt sich nicht «. ${ }^{40}$ Wo Nietzsche Züchtung propagiert, beruft er sich auf das vor-eugenische, noch dem alten semantischen Kontinuum verhaftete Züchtungsprinzip der Veredelung qua Inokulation. Unter dem Stichwort »Veredelung durch Entartung« heißt es: "Geistiger Fortschritt eines Gemeinwesens hängt an den moralisch Schwächeren. An einer Wunde wird etwas neues inokuliert, aber das Ganze muß stark genug sein, um es aufzunehmen. ${ }^{41}$ Dieser erziehenden Veredelung ist

37 Friedrich Nietzsche, Götzen-Dämmerung, in: KSA 3, 96.

38 Vgl. Gerd Schank, Rasse und Züchtung bei Nietzsche, Berlin/New York 2000.

39 Allerdings teilt Nietzsche mit den Eugenikern die Vorstellung einer physiologisch-kulturellen Dekadenz. Vgl. Peter Weingart u.a. (Hg.), Rasse. Blut und Gene. Geschichte der Eugenik und Rassenhygiene in Deutschland, Frankfurt a.M. 1988, S. 84ff.

40 Friedrich Nietzsche, Nachgelassene Fragmente 1887-1889, in: Ders., KSA 13, S. 315.

41 Ders., Menschliches, Allzumenschliches, 5. Hauptstück, Nr. 224, in: Ders., KSA 2, S. $187 f$. Ohne Nietzsche zu nennen, knüpfen jüngste Versuche, Trauma nicht einseitig als Zusam- 
auch Nietzsches individuelles Erziehungsprogramm für potentielle Genies verpflichtet:

Bei dem einzelnen Menschen lautet die Aufgabe der Erziehung so: ihn so fest und sicher hinzustellen, dass er als Ganzes gar nicht mehr aus seiner Bahn abgelenkt werden kann. Dann aber hat der Erzieher ihm Wunden beizubringen oder die Wunden, welche das Schicksal ihm schlägt, zu benutzen, und wenn so der Schmerz und das Bedürfniss entstanden sind, so kann auch in die verwundeten Stellen etwas Neues und Edles inoculiert werden. Seine gesammte Natur wird es in sich hineinnehmen und später, in ihren Früchten, die Veredelung spüren lassen. ${ }^{42}$

Weil der Mensch für Nietzsche das noch nicht festgestellte Tier ist, besteht die Aufgabe der Erziehung darin, ihn so fest hinzustellen, daß er stark genug ist, die veredelnden Traumata zu ertragen. Als Veredelungsprozeß ist Züchtung für Nietzsche eine mögliche Form der Erziehung.

Er äußert sich hier aber nicht über den primären, der veredelnden Verletzung vorangehenden Erziehungsprozeß. Was ist nötig und wie ist es möglich, den Zögling so hinzustellen, daß ihn nichts aus seiner Bahn wirft und er reif wird, nicht für die Autonomie, sondern für schmerzliche Verwundungen seiner bereits erreichten Stabilität? Nietzsches Antwort ist so überraschend wie im Licht seiner Erkenntnistheorie konsequent: "Was ist Erziehung? Daß man sofort alles Erlebte unter bestimmten Wahnvorstellungen begreift. Der Werth dieser Vorstellungen bestimmt den Werth der Bildungen und Erziehungen. In diesem Sinne ist Erziehung Intellekt-Sache, somit bis zu einem Grade wirklich möglich.«" Die intellektuelle Wahnvorstellung, es gäbe Erziehung, ist der Ermöglichungsgrund von Erziehung. Als Wahn ist Erziehung aber nicht zu entlarven, sondern zu rechtfertigen. Dann gibt es sie für Nietzsche so "wie eine Freiheit des Willens - nämlich als nothwendige Wahnvorstellung, als vorgeschobnen Erklärungsgrund für ein uns gänzlich entzogenes Phänomen« ${ }^{44}$ Nietzsche glaubt nicht an die Macht der Erzie-

menbruch der Erinnerung, sondern als stabilisierenden Faktor zu begreifen, mittelbar an Nietzsche an. Vgl. Aleida Assmann, Stabilisatoren der Erinnerung - Affekt, Symbol, Trauma, in: Jörn Rüsen/Jürgen Straub (Hg.), Die dunkle Spur der Vergangenheit. Psychoanalytische Zugänge zum Geschichtsbewußtsein. Erinnerung, Geschichte, Identität 2, Frankfurt a.M. 1998, S. 131-152 sowie Fritz Breithaupt, The Invention of Trauma in German Romanticism, in: Critical Inquiry 32 (2005), S. 77-101.

42 Friedrich Nietzsche, ebd., S. 188.

43 Ders., Nachgelassene Fragmente 1869-1874, in: Ders., KSA 7, S. 122.

44 Ebd., 129f. 
hung, aber er glaubt an die Macht des Glaubens an Erziehung, denn: "alle Staaten und Ordnungen der Gesellschaft: die Stände, die Ehe, die Erziehung, das Recht, alles diess hat seine Kraft und Dauer allein in dem Glauben der gebundenen Geister an sie. ${ }^{45}$ Der Erziehungsprozeß besteht folglich nicht in der Aufklärung über die Wahnvorstellung, sondern in ihrer Automatisierung, denn "es ist das Werk aller Erziehung bewußte Thätigkeiten in mehr oder weniger unbewußte umzubilden. ${ }^{46}$ Für Erzieher und Zöglinge gleichermaßen gilt weiterhin, daß Erziehung "also Bestimmbarkeit des Charakters, freie Wahlentschließung usw. voraus(setzt) - für die Praxis, leugnet aber theoretisch dieselbe und stellt dies Problem sofort an die Spitze der Erziehung. ${ }^{47}$ Das Wissen um ihren Wahn macht Erziehung nicht wirkungslos, sondern kann unter Umständen auch zum Inhalt von Erziehung werden. Erziehung zum Selbstdenker oder Genius "heißt, das Illusionsnetz nothwendig zu machen, durch eifrige Betrachtung des Widerspruchs. $\aleph^{48}$ Aufklärung, die nur über die Irrtümer aufklärt, aber nicht über deren Notwendigkeit, begibt sich nach Nietzsche der großen Vernunft, die in aller Erziehung zu einer Moral waltete, die ihre Zöglinge mit der Sicherheit eines Instinktes auszustatten wußte. Bei aller Kritik, die er an den Weltverbesserern seit dem Christentum übt, erkennt er den Versuch, zu quasi-instinktivem Verhalten zu erziehen, als Verdienst an, »daß man hier die Sicherheit eines Instinkts zu erreichen suchte: so daß weder die gute Absicht, noch die guten Mittel als solche erst ins Bewußtsein traten. So wie der Soldat exercirt, so sollte der Mensch handeln lernen. In der That gehört dieses Unbewußtsein zu jeder Art von Vollkommenheit«. ${ }^{49}$ Weil seit Platon eine "Entnatürlichung« der Moralwerte stattgefunden habe $e^{50}$, ist die Anerziehung von Instinkten um so wichtiger. Der bürgerliche Bildungsbegriff verlangt hingegen umgekehrt die Entlassung der Zöglinge in eine vermeintliche Selbständigkeit, bevor sie festgestellt, bevor sie Instinkte gelernt, bevor ihnen etwas in Fleisch und Blut übergegangen ist.

Das Beispiel des soldatischen Exerzierens suggeriert, daß Nietzsche hinsichtlich der Methodik solcher Instinkt- und Veredelungs-Erziehung, an humanistischer Pädagogik und moderner Disziplinarmacht vorbei, auf das

45 Ders., Menschliches, Allzumenschliches I, in: Ders., KSA 2, S. 191.

46 Ders., Nachgelassene Fragmente 1875-1879, in: Ders., KSA 8, S. 62.

47 Ders., Nachgelassene Fragmente 1869-1874, in: Ders., KSA 7, S. $130 f$.

48 Ebd.

49 Ders., Nachgelassene Fragmente 1887-1889, in: Ders., KSA 13, S. 288.

50 Ebd. 
Arsenal vormoderner Züchtigungstechniken zurückzugreift. Allerdings sind sie nicht Mittel auf dem Weg der zähmenden Zurüstung des Individuums, sondern stehen umgekehrt im Dienst der Herstellung von Instinkten zweiter Ordnung. Dieser Unterschied betrifft nicht nur die Ziele, sondern auch die Verfahren. Während man die Ausnahme durch »eifrige Betrachtung" des Widerspruchs aller Erziehung zum Selbst-Denker erzieht, möchte Nietzsche die allgemeine Bildung weder dem Begriff noch der Prügelstrafe anvertrauen. Hauptsache sei vielmehr »die Zucht nach Mustern. Die Bildung ist nicht nothwendig eine begriffliche, sondern vor allem eine anschauende und richtig wählende: wie der Musiker richtig im Finstern greift. Die Erziehung des Volkes zur Bildung ist wesentlich Gewöhnung an gute Vorbilder «. ${ }^{51}$ Nietzsche rehabilitiert nicht vormodernes Strafen, sondern die Nachahmung und erinnert damit an eine früher selbstverständliche Dimension der Erziehung, die über dem schlechten Gewissen, das seit der Aufklärung auf aller Pädagogik und allem Erziehen lastet, in Vergessenheit geraten ist. Eine als Nachahmung von Vorbildern begriffene Erziehung ist keine Herrschaftstechnik, denn Mimesis ist keine Macht, sondern ein Vermögen. Der moderne Zweifel an Autoritäten ist gewiß gerechtfertigt, aber er betrachtet nur einseitig die Macht des Vorbildes, nicht das Vermögen des Nachahmers. Gewiß billigt Nietzsches Zucht nach Mustern der Erzieher-Person zwar keine Macht, aber doch eine Bedeutsamkeit zu, von der in der Moderne vielleicht nur noch die Psychoanalyse etwas ahnt oder wissen will. ${ }^{52}$ Für Nietzsche werden die erzieherischen Wahnvorstellungen in der Tat »nur durch die Wucht der Persönlichkeiten mitgeteilt « ${ }^{53}$, aber jene "zauberische Einwirkung von Person auf Person«, die sich in der Übertragung der Wahnvorstellungen äußert, läßt sich nicht auf ein übersichtliches Herrschaftsverhältnis abbilden ${ }^{54}$, zumal für Nietzsche bekanntlich gilt: »Wenn man keinen guten Vater hat, so soll man sich einen anschaffen. $\aleph^{55}$ Mimesis lenkt den Blick weg vom Machtverhältnis

51 Ders., Nachgelassene Fragmente 1869-1874, in: Ders., KSA 7, S. 511.

52 Vgl. Shoshana Felman, Psychoanalysis and Education. Teaching Terminable and Unterminable, in: Barbara Johnson (Hg.), The Pedagogical Imperative. Teaching as a Literary Genre (Yale French Studies 63, 1982), S. 21-44.

53 Friedrich Nietzsche, Nachgelassene Fragmente 1869-1874, in: Ders., KSA 7, S. 122.

54 Es zeugt einmal mehr von einer oft frappierenden Übereinstimmung zwischen Nietzsche und Freud, daß Nietzsche an dieser Stelle ausgerechnet den späteren psychoanalytischen Begriff der Übertragung wählt. Zur vergessenen Macht des mimetischen Vermögens vgl. im Anschluß an Walter Benjamin auch Michael Taussig: Mimesis and Alterity. A Popular History of the Senses, New York/London 1993.

55 Friedrich Nietzsche, Menschliches, Allzumenschliches, in: Ders., KSA 2, S. 266. 
zwischen Erzieher und Zögling und erschließt der Erziehung ein anderes Medium. Bei dem, was Sloterdijk "Domestikationsgeheimnis" nennt und Nietzsche das allen Erziehern, Zöglingen und Erziehungstheoretikern »entzogene Phänomen«, für das Erziehung ein "vorgeschobner Erklärungsgrund« sei, könnte es sich um jene "zauberische Einwirkung von Person auf Person« handeln.

Nietzsches Einsatz für Mimesis als Medium erzieherischer Ereignisse und Abenteuer schließt bruchlos an seine ästhetischen Prioritäten an. Bekanntlich behauptete er in dem berühmten Aphorismus über Die Revolution in der Poesie, daß der französischen Literatur seit Voltaire und der deutschen seit Goethe nichts gelungen sei als "haltloses Experimentieren « ${ }^{56}$ Auf die "Querelle" und damit auf den Verlust verbindlicher Muster und die Preisgabe des Nachahmungspostulates, also auf den Beginn der autonomen Kunst, datiert Nietzsche den Beginn des Kunstzerfalls. Als parallele Folgeerscheinungen der verworfenen Rhetorik verbindet Pädagogik und Ästhetik ihre gemeinsame Tabuisierung mimetischer Impulse.

Nietzsches subjektivem literarischen Geschmack zum Trotz machte die literarische Probe auf sein Exempel von der Erziehung als mimetische Übertragung von Wahnvorstellungen ein moderner Autor, der im übrigen mit seiner Erziehung vielfach haderte: »Wenn ich es bedenke, so muß ich sagen, daß mir meine Erziehung in mancher Richtung sehr geschadet hat. ${ }^{57}$ Ausgerechnet Kafka, bei dem Macht und Ohnmacht der Erziehung ineinander übergehen, hat den literarischen Kommentar zu Nietzsches Erziehung durch Mimesis verfaßt. In seinem Bericht für eine Akademie vollzieht sich die Erziehung des »entnatürlichten" Affen nicht als vormoderne Dressur der Art, die an dem Affen Peter und, auf andere Weise, an der halbdressierten Schimpansin mit dem irren Blick verbrochen wurde. Für den sich selbst erziehenden Rotpeter wird Nachahmung zum Ausweg, zum "Menschenausweg ${ }^{58}$ aus der ausweglosen Lage, »immer vorausgesetzt, daß nicht die Freiheit zu wäh-

56 Ders., Menschliches, Allzumenschliches, in: Ders., KSA 2, S. $180 f$.

${ }^{57}$ Franz Kafka, Tagebücher, in: Ders., Schriften. Tagebücher, hg. von Gerhard Neumann u.a., Frankfurt a.M. 2002, S. 17. Vgl. Thomas Schestag, "Dach« [Zu Kafkas Theorie der Erziehung] in: Einfache Lösungen. Beiträge zur beginnenden Unvorstellbarkeit von Gesellschaft, hg. von Bernd Ternes u.a., Würzburg 2000, S. 108-155.

58 Franz Kafka, Ein Bericht für eine Akademie, in: Ders., Schriften. Tagebücher. Kritische Ausgabe, hg. von Gerhard Neumann u.a., Frankfurt a.M. 2002, S. 299-313, hier S. 312. Zur Mimesis bei Kafka vgl. Gerhard Neumann: »Ein Bericht für eine Akademie.« Erwägungen zum »Mimesis« Charakter Kafkascher Texte, in: DVjs 49 (1975), S. 166-183. 
len war « ${ }^{59}$, vorausgesetzt also, daß Freiheit und Autonomie keine Gegenstände einer Wahl sein können, weil sie die zu erlangende Freiheit bereits voraussetzen. Der Plan zur Nachahmung wurde folglich auch nicht aufgrund von Aussichten auf eine gebildete Zukunft oder Versprechungen von Freiheit gefaßt, »daß, wenn ich so wie sie werden würde, das Gitter aufgezogen werde «. ${ }^{60}$ Dieses Tier trieb kein Verlangen, ein Mensch zu werden. Was ihn schließlich zur Nachahmung drängt, ist nichts als akkumulierte und zusehends faszinierte Beobachtung. Sie zeigt "von selbst in die bestimmte Richtung. Es war so leicht, die Leute nachzuahmen ${ }^{6 .}{ }^{61}$

Angesichts von immer früher eingeforderter Freiheit und Selbständigkeit, die heute auch gern als Flexibilität firmiert, könnte eine Besinnung auf die mimetische Dimension in allen Erziehungskommunikationen doppelt hilfreich sein. Zum einen würde dadurch die Erziehung von ihrer furchterregenden Macht etwas entlastet, ohne sie doch deshalb verwerfen zu müssen; zum anderen wüchse durch die Erinnerung an das mimetische Vermögen den vermeintlich austauschbar gewordenen Inhalten eine Legitimität zu, die manch einer wünschen mag, ohne dafür andere Gründe als Sentimentalität namhaft machen zu können.

Gegenwärtig hätte ein entsprechender Kanon Nietzsches Erziehungsreflexionen und Kafkas Affen in jedem Fall einzuschließen. Die einen, weil ihr Verfasser an Erziehung als (notwendiger) Wahnvorstellung festhielt, den anderen, weil er diesen Wahnsinn derart eifrig betrieb, daß sein Lehrer "selbst davon fast äffisch wurde, bald den Unterricht aufgeben und in eine Heilanstalt gebracht werden mußte. Glücklicherweise kam er bald wieder hervor. $\ll^{62}$

Ebd.

Ebd., S. 307.

Ebd., S. 308.

Ebd., S. 312. 


\section{Literaturverzeichnis}

Theodor W. Adorno, Erziehung zur Mündigkeit. Vorträge und Gespräche mit Hellmut Becker, 1959-1969, hg. von Gerd Kadelbach, Frankfurt a.M. 1970.

Giorgio Agamben, Homo Sacer. Die souveräne Macht und das nackte Leben. Aus dem Italienischen von Hubert Thüring, Frankfurt a.M. 2002.

Louis Althusser, Marxismus und Ideologie, Westberlin 1973.

Aleida Assmann, Stabilisatoren der Erinnerung - Affekt, Symbol, Trauma, in: Jörn Rüsen/Jürgen Straub (Hg.), Die dunkle Spur der Vergangenheit. Psychoanalytische Zugänge zum Geschichtsbewußtsein. Erinnerung, Geschichte, Identität 2, Frankfurt a.M. 1998, S. 131-152.

Dirk Baecker, Erziehung im Medium der Intelligenz, http://homepage.mac.corn/ baecker/, S. 1-45.

Dirk Baecker, Kleine Soziologie der Erziehung. Sechsteilige Serie in der »taz«, Februar/März 2004.

Siegfried Bernfeld, Sysiphos oder die Grenzen der Erziehung, Frankfurt a.M. 1973 (1925).

Fritz Breithaupt, The Invention of Trauma in German Romanticism, in: Critical Inquiry 32 (2005), S. 77-101.

Bernhard Bueb, Lob der Disziplin. Eine Streitschrift, Berlin 2006.

Judith Butler, Überlegungen zur Genese des Gewissens, in: Dies., The Psychic Life of Power. Theories in Subjection, Stanford 1997.

Hans Magnus Enzensberger, Aussichten auf den Bürgerkrieg, Frankfurt a.M. 1993.

Shoshana Felman, Psychoanalysis and Education. Teaching Terminable and Unterminable, in: Barbara Johnson (Hg.), The Pedagogical Imperative. Teaching as a Literary Genre (Yale French Studies 63, 1982), S. 21-44.

Michel Foucault, Überwachen und Strafen. Die Geburt des Gefängnisses, Frankfurt a.M. 1977.

Petra Gehring, Was heißt Biopolitik?, Frankfurt a. M. 2006, S. 154-183.

Eva Geulen, Erziehung als blinder Fleck bei Agamben, in: Texte zur Kunst 53 (2004), S. 104-113.

Ulrich Hermann, "Neue Erziehung. Neue Menschen. « Erziehung und Bildung zwischen Kaiserreich und Diktatur, Weinheim/Basel 1987.

Peter Uwe Hohendahl, Literarische Kultur im Zeitalter des Liberalismus 18301870, München 1985.

Werner Jaeger, Paideia. Die Formung des griechischen Menschen, Berlin/New York 1973, S. 840-846.

Franz Kafka, Schriften. Tagebücher. Kritische Ausgabe, hg. von Gerhard Neumann u.a., Frankfurt a.M. 2002. 
Immanuel Kant, Über Pädagogik, in: Ders., Werke, Akademieausgabe, Bd. IX, Berlin 1968, S. 441-499.

Dieter Lenzen (Hg.), Irritationen des Erziehungssystems. Pädagogische Resonanzen auf Niklas Luhmann, Frankfurt a.M. 2004.

Niklas Luhmann, Das Erziehungssystem der Gesellschaft, Frankfurt a.M. 2002.

Jan Masschelein/Maarten Simons, Globale Immunität oder Eine kleine Kartographie des europäischen Bildungsraums, Zürich/Berlin 2005.

Käte Meyer-Drawe, Erziehung und Macht, in: Vierteljahrsschrift für Wissenschaftliche Pädagogik 77/4 (2001), S. 446-457.

Käte Meyer-Drawe, Illusionen von Autonomie. Diesseits von Ohnmacht und Allmacht des Ich, München ${ }^{2} 2000$.

Robert von Mohl, Die Polizei-Wissenschaft nach den Grundsätzen des Rechtsstaats, Tübingen 1833, I.

Ernst Müller (Hg.), Gelegentliche Gedanken über Universitäten, Leipzig 1990.

Gerhard Neumann: „Ein Bericht für eine Akademie.« Erwägungen zum »Mimesis« Charakter Kafkascher Texte, in: DVjs 49 (1975), S. 166-183.

Friedrich Nietzsche, Kritische Studienausgabe in 15 Bden., hg. von Giorgio Colli und Mazzino Montinari, Berlin/New York 1980.

Talcott Parson/Gerald M. Platt, Die amerikanische Universität. Ein Beitrag zur Soziologie der Erkenntnis, übers. von Michael Bischoff, Frankfurt a.M. 1990.

Jacques Rancière, The Ignorant Schoolmaster, Five Lessons in Intellectual Emancipation, New York 1991.

Markus Rieger-Ladich/Norbert Ricken (Hg.), Michel Foucault. Pädagogische Lektüren, Wiesbaden 2004.

Markus Rieger-Ladich, Mündigkeit als Pathosformel. Überlegungen zur pädagogischen Semantik, Konstanz 2002.

Katharina Rutschky (Hg.), Schwarze Pädagogik. Quellen zur Naturgeschichte der bürgerlichen Erziehung, Frankfurt a.M./Berlin 1988.

Gerd Schank, Rasse und Züchtung bei Nietzsche, Berlin/New York 2000.

Gerhart Schmidt, Nietzsches Bildungskritik, in: Mihailo Djuric/Josef Simon, Zur Aktualität Nietzsches, Würzburg 1984, S. 7-16.

Peter Sloterdijk, Regeln für den Menschenpark. Ein Antwortschreiben zu Heideggers Brief über den Humanismus, Frankfurt a.M. 1999.

Peter Sloterdijk, Zorn und Zeit. Politisch-Psychologischer Versuch, Frankfurt a.M. 2006.

Peter Weingart u.a. (Hg.), Rasse. Blut und Gene. Geschichte der Eugenik und Rassenhygiene in Deutschland, Frankfurt a.M. 1988. 
